\title{
The point mutations in the fingers domain increase the fidelity of DNA synthesis on undamaged DNA and abrogate DNA translesion synthesis in Y-family of DNA polymerases
}

\author{
E.O. Boldinova, N.A. Miropolskaya, A.V. Ignatov, A.V. Kulbachinskiy, \\ A.V. Makarova* \\ Institute of Molecular Genetics RAS, Moscow, Russia \\ *e-mail: amakarova-img@yandex.ru
}

Key words: Y-family of DNA polymerases, Pol iota, Pol eta, DNA damage, active site

\begin{abstract}
Motivation and Aim: Eukaryotic Y-family of DNA polymerases Pol iota and Pol eta play a key role in efficient and accurate replication of DNA with damage (DNA translesion synthesis, TLS) but demonstrate very low fidelity of DNA synthesis on undamaged DNA templates.
\end{abstract}

Methods and Algorithms: To better understand the mechanism of translesion and errorprone DNA synthesis by Pol iota and Pol eta we investigated substitutions of evolutionary conserved active site residues in the fingers domain, involved in interactions with the templating and the incoming nucleotides. We analyzed the efficiency and fidelity of DNA synthesis by the mutant human and yeast polymerase variants opposite thymine dimers, abasic site, thymine glycol, 8-oxoguanine, 1, $\mathrm{N}^{6}$-ethenoadenine and on undamaged DNA. Results: The point amino acid substitutions Y39A and Q59A in human Pol iota and Q55A and R73A mutations in yeast Pol eta decreased the catalytic activity of DNA polymerases on undamaged templates and significantly affected DNA damage bypass. In the presence of $\mathrm{Mg}^{2+}$ ions, the Q59A amino acid substitutions abolished the incorporation of dNTP opposite $1, \mathrm{~N}^{6}$-ethenoadenine by Pol iota suggesting that the residue Glu59 plays a role in the Hoogsteen base pairs formation during catalysis. The Q55A substitution in Pol eta significantly reduced the efficiency of thymine dimers bypass, R73A had a stronger effect on the activity opposite abasic site, while both substitutions impaired replication opposite thymine glycol. Importantly, Y39A and Q59A mutations in human Pol iota and R73A and, to a lesser extent, Q55A substitutions in yeast Pol eta increased the fidelity of DNA synthesis opposite undamaged DNA.

Conclusions: Altogether, these results reveal a key role of conservative residues of the fingers domain of Y-family DNA polymerases in replication opposite various types of DNA lesions and highlight the evolutionary importance of TLS function at the cost of accuracy of DNA replication.

Acknowledgments: This work was supported by RFBR grant No. 17-00-00264 and the RAS program "Molecular Biology. New groups". 